\title{
Preface
}

\section{XIlth Quark Confinement and the Hadron Spectrum}

This publication of the proceedings of the XIlth Conference on Quark Confinement and the Hadron Spectrum is a collection of papers selected and presented at plenary, parallel and poster sessions.

The Quark Confinement conference series aim is to bring together people working in QCD and strong-interaction dynamics. This edition of the conference took place in Thessaloniki (Greece) from 29th August to 3rd September 2016 in Makedonia Palace conference centre (https://indico.cern.ch/e/conf12). It was dedicated to the memory of Michael Müller-Preußker. The conference has also included a variety of events addressing school children and general public as well as different cultural events open to the public.

The following topics were covered at the XII Conference:

Vacuum Structure and Confinement:

Mechanisms of quark confinement (vortices, monopoles, calorons...) and the structure of the vacuum in non-Abelian gauge theories. Chiral symmetry breaking, and the Dirac spectrum in the low-momentum region. Studies of ghost and gluon propagators. Confining strings and flux tubes, their effective actions. Renormalons and power corrections. Interface between perturbative and non-perturbative physics.

Focus Subsection: Emergent gauge fields and chiral fermions.

\section{Light Quarks:}

Chiral and soft collinear effective theories; sum rules; lattice; SchwingerDyson equations; masses of light quarks; light-quark loops; phenomenology of light-hadron form factors, spectra and decays; structure functions and generalized parton distributions; exotics and glueballs; experiments.

Heavy Quarks:

Heavy-light mesons, heavy quarkonia, heavy baryons, heavy exotics and related topics: phenomenology of spectra, decays, and production; effective theories for heavy quarks (HQET, NRQCD, PNRQCD, VNRQCD, SCET); sum rules for heavy hadrons; lattice calculations of heavy hadrons; heavy-quark masses determination; experiments. 
Deconfinement:

QCD at finite temperature; quark-gluon plasma detection and characteristics; jet quenching; transportation coefficients; lattice QCD and phases of quark matter; QCD vacuum and strong fields; heavy-ion experiments.

QCD and New Physics:

Physics beyond the Standard Model with hadronic physics precision experimental data and precision calculations.

Nuclear and Astroparticle Physics:

Nuclear matter; nuclear forces; quark matter; neutron and compact stars.

Strongly Coupled Theories:

Hints on the confinement/deconfinement mechanisms from supersymmetric and string theories; strongly coupled theories beyond the Standard Model; applications of nonperturbative methods of QCD to other fields.

Statistical Methods for Physics Analysis in the XXI Century:

Machine learning techniques; data fitting and extraction of signals; new developments in unfolding methods; averaging and combination of results.

Upgrades and Instrumentation:

Probing QCD and facilities, future experiments, planned upgrades, performance studies, simulation and analysis methods, instrumentation and new technologies.

The conference was followed by a Workshop on Accelerators Revealing the QCD Secrets devoted to the progress, current status and open challenges of QCD-driven physics.

We would like to thank all the conference participants as well as the members of the International Advisory Committee, the Local Organizing Committee, Assistant students, the Organizing Institutes and Sponsors.

\section{Yiota Foka, Nora Brambilla, Vladimir Kovalenko}

\section{Editors}




\section{The XIlth Quark Confinement and the Hadron Spectrum Conference $^{1}$}

The conference series "Quark Confinement and the Hadron Spectrum", inaugurated in 1994 by Giovanni Prosperi and Nora Brambilla initially with strong focus on theory aspects, has been well established by now as an important forum expanding on all aspects of strong interaction physics and attracting theoretical and experimental physicists alike.

The XII edition of the conference was held in Thessaloniki, Greece, from the 29th of August to the 3rd of September 2016, organized by ALICE's own Yiota Foka, co-chaired by Nora Brambilla and with the assistance of a diverse international Organizing Committee. It attracted about 400 participants, ranging from students to world experts that enjoyed a rich scientific programme and was hosted in the beautiful venue of Makedonia Palace conference centre. It provided an ideal setting for intellectual debates, situated in the heart of a dynamic modern city with a history of more than 2500 years. The conference coincided with the year celebrating Aristotle, who had his school in the area and was teaching Alexander the Great among others.

Quark confinement, a striking fundamental phenomenon that prevents colour charged particles from being isolated singularly, was the general point at issue of the conference.

Different aspects of strong interactions physics and related fields were covered by 40 invited plenary overview presentations complemented by 267 parallel talks and 33 posters. World leading scientists reported on the most up-to-date topics of interest and discussed a variety of open issues. The complexity and diversity of QCD, the theory of strong interactions, as well as its relevance to other related fields, were reflected by seven parallel sections. They addressed current theoretical developments and recent experimental results covering topics from typical QCD theory to nuclear and astroparticle physics and searches for new physics.

This edition has seen several additions for the first time. The conference was preceded by introductory lectures for students on different topics of QCD also highlighting general relativity, gravitational waves and the recent discovery of LIGO-VIRGO experiments.

${ }^{1}$ Published in ALICE Matters (C) 2016 CERN on behalf of the ALICE Experiment http://alicematters.web.cern.ch/?q=content/node/986 
The conference was further expanded with an extra plenary session on Saturday morning, addressing Future Perspectives and summarizing the main experimental and theoretical developments and directions of the field.

In addition, newly included parallel sessions enriched the programme with presentations on "Statistical Methods for Physics Analysis" organized by Tommaso Dorigo of CMS, as well as on "Upgrades and Instrumentation" organized by Pasquale Di Nezza of ALICE and Christian Sturm of CBM. Both sessions were well attended and provided a link between theoretical ideas and the challenges of reality addressed by experimentalists who work in the data analysis front and push detector technology frontiers.

Several round-table discussions stimulated scientific debates and were complemented by lively conversations between participants enjoying the views to the Mediterranean Sea and breath-taking sunsets during the coffee breaks.

Another novelty of this edition: the authors of five selected best posters were awarded invitations for flash talks in the plenary session allowing them to give a brief summary of their research. In general the newer generation has taken its share in the conference timetable with numerous contributions by young researchers.

Outreach also had its space with a report in the plenary session. The presentation of the rich outreach activities realized in Greece in the last years encouraged participants to further carry out analogous activities with the aim of raising students' interest in science.

Sparkling humour was introduced by the closing talk of Alvaro De Rújula who amused the participants presenting "the main aspects of QCD in its infancy" in his talk entitled "The November Revolution".

A further particular twist was given with the addition of the workshop "Accelerators revealing the QCD secrets" which followed the conference, 35 September 2016.

The workshop, organized by Yiota Foka and Oliver Kester, of GSI, and Emmanuel Tsesmelis, of CERN, focused on the requirements for future accelerators for QCD studies. It was attended by a mixed community of theorists, experimentalists and machine leading experts, who enjoyed the innovative interesting agenda with various topics spanning "from theory to reality" and had the opportunity to exchange views on future developments and directions. The Saturday afternoon session celebrated the $70^{\text {th }}$ anniversary of Peter Braun-Munzinger, the Chair of the ALICE Collaboration 
Board. Some 60 participants joined a boat trip and the birthday dinner by the beach.

\section{Outreach events}

The scientific programme was complemented by outreach events for the general public intended to show the societal benefits of fundamental research. The weekend before the start-up of the conference included Science Events in the City Hall of Thessaloniki and the Public Library of the neighboring town Veroia. A whole day programme of science activities, addressing public of all ages included presentations and discussions, exhibitions and projections, simple physics experiments. Moreover, participants had the possibility to perform analysis of real data and to "discover" the Higgs boson as it was done by physicists at the Large Hadron Collider (LHC) at CERN. The public talks in Greek by Prof. Emmanuel Tsesmelis were followed by vivid discussions with the public and were complemented by really exciting musical performance by soprano Kalliopi Petrou, accompanied at the piano by our CMS colleague Tommaso Dorigo.

The conference participants also had the opportunity to enjoy, a few days later, another brilliant performance of Kalliopi in an Opera Gala event. Numerous other social events paralleled the tight scientific programme highlighting the history and culture of the area. A memorable experience was the visit to the stunning archeological complex of the Macedonian tombs including the one of King Philip II, father of Alexander the Great, followed by an enjoyable dinner with traditional music and dancing. Participants also appreciated a visit to Aristotle's school and a lecture on his influence in science hosted by the "Aristotle University of Thessaloniki. The conference dinner at a beach bar gave the opportunity for a swim before a treat with Russian caviar, offered by the Russian members of the Organising Committee, and a traditional Greek meal and dancing performance.

The conference was possible thanks to financial and in-kind contributions of numerous sponsors and the valuable assistance of the Organising Committee. It is worth mentioning the remarkably effective cooperation of the core-team young members that worked remotely on all details of the conference organization from different parts of the world, spanning from Greece and CERN to Poland, Russia and Spain. Integrating in place the assistant students from Greek universities, they ensured the smooth running of the conference and had the pleasure of receiving the welldeserved enthusiastic comments from numerous participants appreciating the unforgettable experience. 


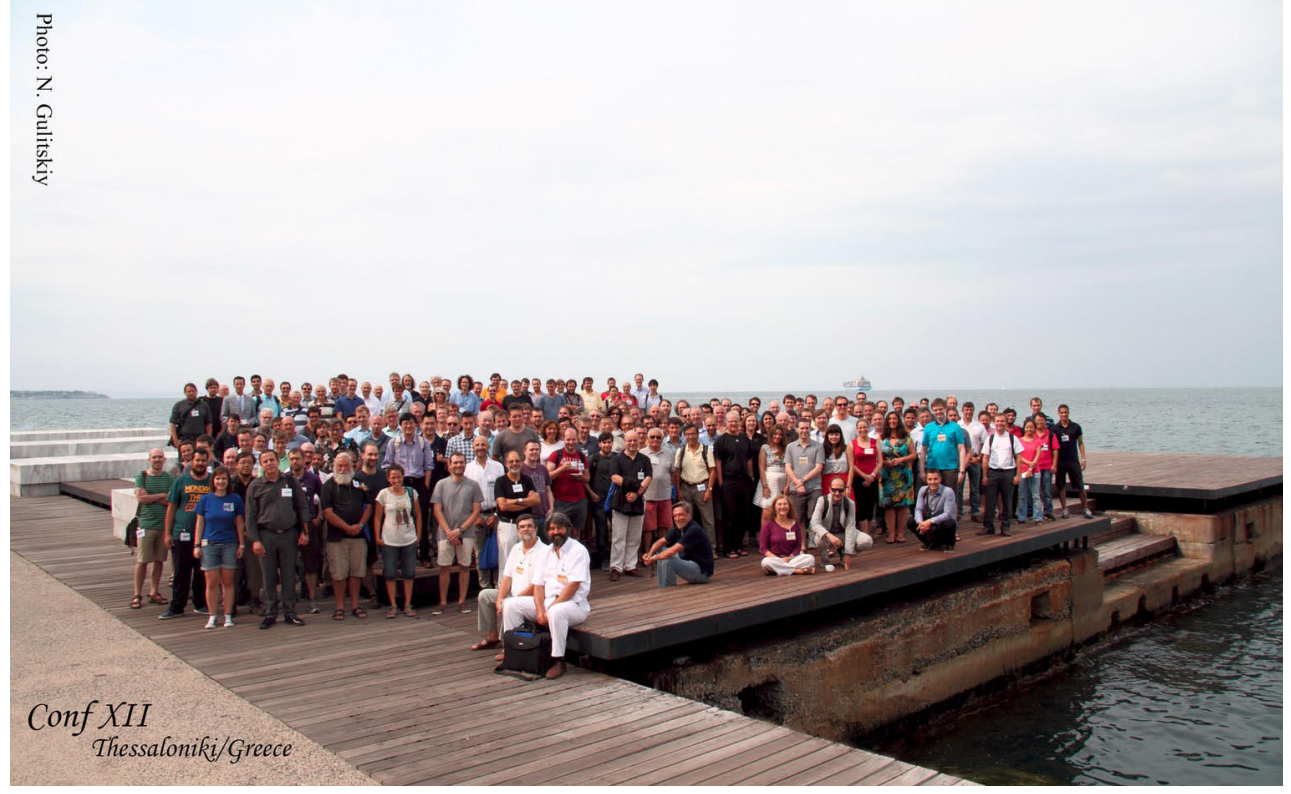

Quark Confinement XII Group photo.

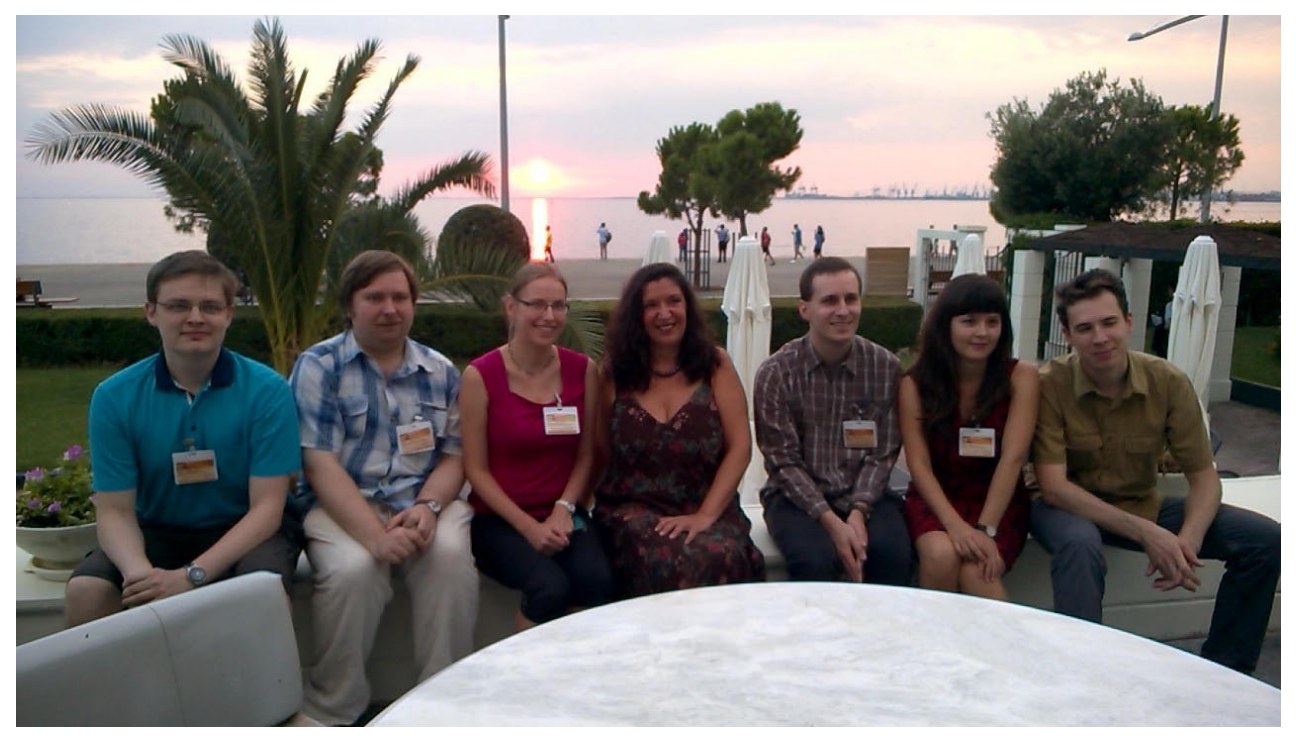

Core team of the young members of the organizing committee: E. Andronov (SPbSU), V. Kovalenko (SPbSU), M. Janik (WUT), L. Graczykowski (WUT), A. Katanaeva (SPbSU) and N. Gulitsky (SPbSU). 